\title{
An Extended Reinforcement Learning Framework to Model Cognitive Development with Enactive Pattern Representation
}

\author{
Zhenping Xie, Yaochu Jin, Fellow IEEE
}

\begin{abstract}
In order to make machines more intelligent, it is inevitable to understand human-like cognitive development, in which adaptive, autonomous and progressive evolution of cognitive decision-making in interacting with the environment plays a key role. Inspired by enactive artificial intelligence and evolutionary sampling learning, a new cognitive development learning model termed evolutionary enactive learning is proposed in this work. The proposed model is constructed by extending the reinforcement learning framework and introducing the utility-selection theory to guide the coevolution of pattern representation and decision-making policies. Theoretical analysis on the model's validity of evolutionary enactive learning is given. To further demonstrate the effectiveness of the proposed method, two simulated cognitive decision-making tasks are designed, in which pattern representation and decision-making must be jointly developed to achieve good cognitive performance. Our experimental results clearly demonstrate that the resulting learning process is rational and effective. Finally, we indicate that the proposed evolutionary enactive learning could be readily further extended by introducing existing machine learning techniques to solve more practical applications.
\end{abstract}

Keywords: Enactive artificial intelligence; cognitive development; pattern representation; evolutionary utility-selection; cognitive decision-making.

\section{INTRODUCTION}

$\mathrm{R}$ ecently, research on computational modeling of human-like intelligence have been attracting increasing attention in the field of artificial intelligence $[1,2,3]$. In such models, decision-making and performing suitable behaviors by properly recognizing the state of the agent itself and environment [4,5] is a fundamental. Meanwhile, modeling the cognitive development process, in particular how machines can autonomously develop their cognitive decision abilities, is crucial for understanding human intelligence [6-10]. In general, an effective cognitive decision system should comprise two key components: an efficient sensory-state pattern representation model and a good decision-making model $[11,12]$.

Over the past 30 years, reinforcement learning (RL) has become the most basic way for achieving autonomous decision-making capabilities in artificial systems $[13,14,15]$. Traditional reinforcement learning methods mainly focus on how to obtain a convergent reward value-function with respect to a set of decision policies [15]. Accordingly, the learnt reward value-function for state-action pairs will be used as a prior experience to choose the optimal action for a future perception state. However, how to construct

- ZP Xie is with the School of Digital Media, Jiangnan University, Wuxi, Jiangsu, 214122 China, and Jiangsu Key Laboratory of Media Design and Software Technology, Wuxi, Jiangsu, 214122 China. E-mail: xiezhenping@hotmail.com.

- Y Jin is with the Department of Computer Science, University of Surrey Guildford, GU2 7XH, UK. E-mail: yaochu.jin@surrey.ac.uk

(Corresponding Author) efficient sensory state pattern representation, and how to define optimal decision policies model must be separately considered by means of other machine learning methods. For example, a Gaussian multilayer network is employed to manually integrate the state-action policies and the corresponding value function in [14] for the car-pole balancing problem. In [11,12], deep convolutional neural networks [16] are adopted to efficiently represent high-dimensional sensory inputs and the value function of a massive number of decision policies. However, these predefined black-box representation structures cannot be adapted to satisfy with the requirements for explicitly understanding the environmental states and performing sufficiently robust decision-making like the human brain.

According to the viewpoints of enactive artificial intelligence, the constitutive autonomy and adaptivity are necessary for the cognitive development of human life [17]. Here, the constitutive autonomy and adaptivity must contain the variability of pattern representation structures of sensory-states and decision-making policies. To imitate the above-mentioned characteristics of human cognitive development, evolutionary enactive learning (EEL) is put forward in this study by extending the reinforcement learning framework. In EEL, an extra utility probability value is allocated to each possible pattern representation item and decision policy item, where utility probability values indicate the statistical usefulness of all possible items according to historical experience. Then, the utility probability values can be used to select the most useful memory items, which is different from the value function used in conventional reinforcement learning. Moreover, 
evolutionary exploration and selective memory can be implemented to evolve state pattern representation and decision policies by means of integrating a utility-selection strategy into the evolutionary sampling learning method.

Similar to considerations in $[11,12]$, the core design of EEL is to close the loop between the objectives of machine learning and action rewards. In deep RL [11] and alphaGo [12], action rewards are converted into an expectation value function, and the learning objective is defined as the optimal approximation to the value function using deep neural network. In EEL, not only reward value functions but also utility probability value functions are designed. Here, the definitions of utility probability value functions conjointly inherit from the ideas of statistical machine learning and reinforcement learning. Thus, machines may carry out more autonomous statistical learning guided by cognitive action rewards from the surviving environment instead of direct supervised learning [18], which should be pivotal for creating more autonomous and robust artificial intelligence systems.

The rest of the paper is organized as follows. In Section II, related machine learning methods are briefly introduced. In Section III the implementation of evolutionary enactive learning algorithm and some theoretical analysis are described. In Section IV, experimental results on two simulated cognitive decision-making games are presented and analyzed. Finally, in Section V some conclusions of this paper are provided.

\section{RELATED LEARNING METHODS}

This work mainly explores the coevolution of cognitive decision-making capabilities along with enactive pattern representation driven by individual actions under environmental constraints, which has so far not yet been studied in the literatures. Even so, some aspects of questions have been touched upon in several machine learning methods. In the following, we will briefly introduce and discuss these methods.

\section{A. Deep reinforcement learning}

For the decision-making process to be adapted to complex input states like those in Atari games, deep reinforcement learning method was put forward in [11]. There, deep neural network modeling and Q-learning are seamlessly integrated into a unified learning framework. In the framework, two key strategies are designed: 1) a batch of latest state-action-reward tuples $\left(s_{t}, a_{t}, r_{t}\right)$ are considered as the updating training objective of deep neural network; 2) current decision-making system is viewed as the optimal representation of past experience, and new experiences are gathered by autonomously exploring and exploiting possible actions and corresponding environmental rewards. In deep RL, the Q-value function in reinforcement learning

$$
\hat{Q}(s, a) \leftarrow \hat{Q}(s, a)+\alpha\left(r+\gamma \max _{a^{\prime}} \hat{Q}\left(s^{\prime}, a^{\prime}\right)-\hat{Q}(s, a)\right)
$$

is replaced as follows:

$$
Q(\phi(s), a ; \theta) \leftarrow r+\gamma \max Q\left(\phi\left(s^{\prime}\right), a^{\prime} ; \theta^{-}\right),
$$

where $s^{\prime}$ is the subsequent state of $s$ after action $a$ is executed, $\phi(\bullet)$ refers to a pre-transform for primitive system states, and $\theta^{-}, \theta$ represent the parameters of deep neural networks on consecutive stages. Specifically, the updating approximation objective can be defined to optimize $\theta$ by collecting a batch of latest $Q\left(\phi\left(s_{j}\right), a_{j} ; \theta^{-}\right)$.

Although deep reinforcement learning can progressively develop and optimize individual decision-making in cases where the input states are high-dimensional, it could only support a limited number of actions. That is, deep reinforcement learning may fail to scale up to a huge number of possible actions as seen in human life. In alphaGo [12], extra policy networks and Monte Carlo tree search strategy are introduced to cope with decision evaluation in the presence of huge possible actions. Even so, explicit pattern and policy representation still cannot be realized.

In this study, a new mechanism, termed utility probability based evolutionary selection learning strategy is introduced to support the coevolution of explicit pattern and policy representation learning. We consider that the environmental rewards for an agent's actions could be converted into utility probability values for related pattern representation and decision policies. Thus, all learning and optimization objectives can be defined by transforming, distributing, and propagating utility probability values.

\section{B. Evolutionary optimization}

Evolutionary computation $[19,20]$ is a powerful tool for optimization in control systems [7,21,22], machine learning $[16,23,24]$, evolutionary robotics [25], and many other applications [26-27]. In general, optimization problems can be described as the following form.

$$
\text { Minimize } f(X) \text {, s.t. } X \in S \subseteq R^{D}
$$

where $f(X)$ is an evaluable function, $S$ is the corresponding decision space of dimension $D$. For an cognitive learning problem, the objective is to seek optimal actions or action probabilities from all possible actions for all possible observed inputs. Here, the dimension $D$ should equal to the number of possible inputs multiplied by the dimension of the action space, which may become very large. Conventional evolutionary optimization methods may become less effective because only a limited number of fitness evaluations may be allowed. To address this issue, special evolutionary optimization strategies should be designed for the evolutionary optimization of cognitive development learning, e.g., surrogate-assisted evolutionary optimization [28].

\section{Pattern analysis}

Pattern analysis $[29,30]$ is a fundamental element of human cognitive capabilities, mainly consisting of pattern representation and pattern classification [31]. Pattern representation $[32,33,34,35]$ refers the way how a pattern is described using numerical forms, while pattern classification $[36,37,38]$ performs the task of distinguishing and recognizing different patterns. In general, the classification of an observed datum $x$ can be formally defined as

$$
C_{j}=f_{c}(\phi(x), \theta)
$$


where, $C_{j}$ is the index of the pattern class in pattern domain $C, f_{c}(\bullet)$ is a classification function with parameter $\theta$, and $\phi(\bullet)$ is a pattern representation transform with respect to original physical signals. Moreover, a pattern analysis model should contain pattern feature representation, classification model and learning method of parameter $\theta$.

For pattern feature representation, it is often manually designed $[39,40]$ or extracted using statistical learning methods $[41,42]$. Nowadays, common classification models include decision tree methods [43,44], support vector machines [45,46], and neural networks [16]. In order to train the model parameter $\theta$, unsupervised or supervised learning strategies can be employed [47].

Though large amount of researches have been carried out in past several decades, there are little work on how to develop a type of pattern analysis systems that are able to completely autonomously evolve based on the active interaction with the environment in which the individual is. Recently, deep neural network methods [16,34] offered a possible way of jointly learning pattern representation and classification provided that a huge number of labeled samples are available. Despite this, the huge number of labeled samples must be prepared by hand or other tools. Lately, deep reinforcement learning [11] and alphaGo [12] further improved the learning autonomy by combing reinforcement learning, in which supervision information could be created by consecutively simulating decision actions and getting subsequent environmental rewards.

In this study, an enactive pattern representation strategy is proposed for explicit modeling of complex pattern representation, which is well complementary to neural network modeling.

\section{Evolutionary sampling learning}

In our previous research [48], a novel machine learning approach within a probabilistic framework, termed evolutionary sampling learning (ESL) was put forward. It was shown that ESL can be used to acquire an approximation representation to any point-wise computable probability function. The learning process of ESL can be formulated as

$$
p^{t}\left(\phi(x) ; \theta^{t}\right) \rightarrow \pi^{t}(\phi(x))
$$

for the learning objective $p^{*}\left(\phi(x) ; \theta^{*}\right) \equiv \pi^{*}(\phi(x))$, where $p^{t}\left(\bullet ; \theta^{t}\right)$ is the approximation representation of $\pi^{t}(\phi(x))$ at time $t$, and $\theta^{t}$ is composed of a subset of past perceptual data. When the learning procedure of ESL terminates, there has $\pi^{t \rightarrow \infty}(\phi(x)) \equiv \pi^{*}(\phi(x))$ or $\pi^{t \rightarrow \infty}(\phi(x)) \rightarrow \pi^{*}(\phi(x))$ for different cases.

Interestingly, ESL could be viewed as a dynamic evolutionary process or an online statistical learning process. Therefore, ESL may be widely applicable to problems those can be converted into probability representation learning problems. In this study, we will employ ESL to evolve cognitive system by introducing utility-selection strategy.

\section{EvolutionARY ENACTIVE LEARNING}

This section will first introduce the framework of EEL, followed by the designed details of the evolutionary process in EEL. We then describe the core algorithms used in EEL. Finally, some theoretical discussions on the model are given.

\section{A. Conceptual Framework}

For an autonomously learnable cognitive system and its surviving environment, we suppose that it can continuously perceive two types of information, the environmental states and the internal states of itself. We also assume that a cognitive system may make decision actions that will result in some causal changes to the environmental states and/or internal states. According to the viewpoints of enactive AI [17], there is no other information that could be used in the developmental learning of a fully autonomous cognitive system.

For sake of the convenience, we introduce cognitive space as a concept to represent the integrated system composed of intelligent agents and their surviving environment. Thus, a cognitive space will be a closed system, and the cognitive development of an agent in a cognitive space can be seen as a procedure of continuously executing individual utility-selection. Here, utility-selection goals can include, e.g., the optimization of certain function $[11,12,14]$, statistically optimal prediction on future system states [4] or optimal surviving robustness [6,7]. Though cognitive development may have diverse utility-selection goals, it should be an essential task for cognitive development learning to discover and memorize the intrinsic laws embedded in a cognitive space. Accordingly, the core task of pattern representation learning is to computationally model these intrinsic laws, while the results of decision policy learning reflect personalized utility-selection preference.

The generic framework of EEL is illustrated in Figure 1. The proposed autonomous agent comprises three kinds of memories and five process modules. It evolves its cognitive behaviors by continually interacting with its surviving environment. In addition, a symmetry representation model as a prior experience is introduced to enactively construct explicit pattern and decision policy representation.

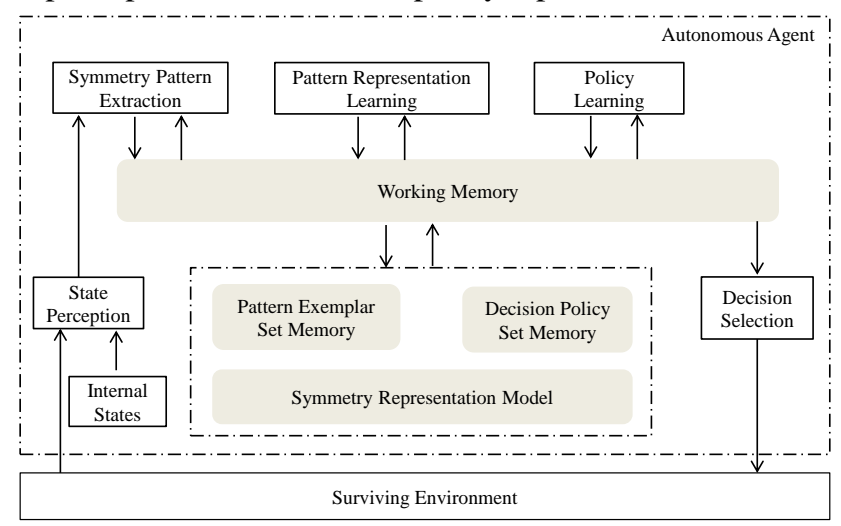

Fig. 1. The framework of evolutionary enactive learning 
In the proposed EEL framework, a symmetry representation model (SRM) is designed to model all possible pattern exemplars of given input sensory-states and decision policies that may be necessary for the agent to fulfill desired autonomous tasks. In SRM, the symmetry representation strategy aims to realize the enactive constitution of explicit pattern representations related to decision policies. To be specific, two types of patterns are considered in SRM, namely, ground patterns and abstract patterns. Here, ground patterns refer to those patterns that can be explicitly represented by means of symmetry information structures on space, time and/or movement positions, which is inspired by the symmetry theory of physical laws in the real world. By contrast, abstract patterns are considered to be represented by a logic combination of ground patterns also with respect to space, time and movement correlation like in our physical world.

Among three types of memories in the EEL framework, working memory is used to store temporary, shared variables required for four process modules as illustrated in Figure 1. In contrast, pattern exemplar set and decision policy set memories are used to store two types of persistent cognitive knowledge data (individual experience), respectively. For five cognitive process modules, their main functions are explained as in Table I.

TABLE I

Five Cognitive FunCTION MODULES IN EEL

\begin{tabular}{ll}
\hline State Perception & $\begin{array}{l}\text { Receiving current sensory signals by } \\
\text { observing individual surviving } \\
\text { environment and the individual itself }\end{array}$
\end{tabular}

\begin{tabular}{ll}
\hline $\begin{array}{l}\text { Symmetry } \\
\text { Pattern }\end{array}$ & $\begin{array}{l}\text { Transforming sensory signals into } \\
\text { formal pattern representation based } \\
\text { Extraction }\end{array}$ \\
\hline Pattern & Learning and memorizing most \\
Representation & useful pattern representation \\
Learning & exemplars by means of the \\
& utility-selection learning strategy \\
\hline Policy Learning & Learning and memorizing most \\
& useful decision policies by means of \\
& the utility-selection learning strategy \\
\hline Decision & Selecting suitable behavior policy to \\
Selection & perform certain actions according to \\
& individual utility-selection preference \\
\hline
\end{tabular}

To explain the role of each component of the proposed EEL framework in Figure 1 in plain language, we take a football player starting to learn playing football as an example. As a beginner, he/she has little memory/skill for playing football. As the player practices playing football, he/she must perceive the environmental and internal states for instance, by observing the positions of the football and players, and experiencing the amount of force when touching the football. The above information is represented by the module "State Perception" in Figure 1. To improve the skills, the player must learn the physical laws governing the movements of the football and the playing strategies or behaviors of other players.
These are denoted by the module "Symmetry Pattern Extraction" and "Pattern Representation Learning". In addition, the module "Policy learning" includes a collection of possible skills for playing football, while the module "Decision Selection" is the decision-making process that aims to maximize the reward. Finally, different memory units are needed, including "Working Memory", "Pattern Exemplar Set Memory" and "Decision Policy Set Memory".

\section{B. Evolutionary process of EEL}

\section{Formal definitions}

According to the above conceptual framework of EEL, some formal definitions will be first provided. Without loss of generality, we use $\left\{x^{t}\right\}_{t \leq T}$ to represent all observable states of a cognitive space within a period of time $T$. Let $x^{t}=\left\{x_{i}^{t}\right\}_{i=1,2, \ldots n}$, where $x_{i}^{t}$ is a subset of $x^{t}$ and different subsets may overlap, and $n$ is the number of all subsets. Similarly, we use $\left\{a^{t}\right\}_{t \leq T}$ to represent all actions performed in the past, and let $a^{t}=\left\{a_{l}^{t}\right\}$, where $a_{l}^{t} \in \mathrm{A}$ and $\mathrm{A}$ is the set of all possible actions. Meanwhile, we use $\left\{r^{t}\right\}_{t \leq T}$ to represent obtained reward from the cognitive space, also $r^{t}$ may contain multiple components. It should be noted that, $r^{t}$ may be invalid or null in some time instants.

Moreover, we use $\Phi$ to denote the set of all possible pattern representation items, where $\Phi=\left\{<\Phi_{k}, u_{k}>\right\}$ and $\Phi_{k}=<\left\{\phi_{j}\left(\left\{x^{t}\right\}_{t \leq T}, \theta_{j}\right)\right\}_{j \in C_{k}}>, \tilde{\phi}=\left\{\phi_{j}\left(\left\{x^{t}\right\}_{t \leq T}, \theta_{j}\right)\right\}_{j \in C}$ is called as the set of ground pattern representation, $u_{k}$ is an utility probability value and $C_{k}$ is a subset of $C$ (the set of all possible ground pattern representation items). We denote the decision policy set as $\mathfrak{R}=\left\{R_{l}=<\Phi_{l}, a_{l}, u_{l}, \hat{r}_{l}>\right\}_{l \in L}$, where $\Phi_{l} \in \Phi, a_{l} \in \mathrm{A}, u_{l}$ and $\hat{r}_{l}$ are the utility probability value and the expectation reward value of decision policy $R_{l}$. The definition of $\hat{r}_{l}$ is similar to the expectation reward value designed in reinforcement learning.

According to above definitions, the learning parameters of a cognitive system will contain pattern representation parameters $\left\{\theta_{l}\right\}$, combination index sets $\left\{C_{k}\right\}$ and decision policy set $\left\{R_{l}\right\}$, if the forms of all possible ground pattern transforms $\left\{\phi_{j}\left(\bullet ; \theta_{j}\right)\right\}$ and all optional actions could be predefined as prior knowledge. It should be noticed that, the decision policy set $\mathfrak{R}=\left\{R_{l}=<\Phi_{l}, a_{l}, u_{l}, \hat{r}_{l}>\right\}_{l \in L}$ could as well be implicitly expressed using a deep neural network as in $[11,12,14]$. Obviously, $\left\{r^{t}\right\}_{t \leq T}$ is the only supervision information that can be used to guide the evolution of an autonomous cognitive system.

In above definitions, two types of pattern representation are considered, one is ground pattern representation, and the other is abstract pattern representation. Ground patterns reflect the basic pattern structures like a corner or a line considered in machine vision, while abstract patterns 
reflect high-level pattern structures like a flower or a house. In an autonomous cognitive system, the above considerations are inevitable [34,49,50]. Furthermore, decision policy set $\left\{R_{l}\right\}$ may be considered as explicit logic reasoning rules [51], interpretable fuzzy rules [52], or representative experience tuples [11]. In addition, utility probability variables $u$ are newly designed for each pattern representation item and each decision policy item. They are used to reflect the relative memory importance of every required memory item in a cognitive system.

\section{Evolutionary learning process of EEL}

As illustrated in Figure 2, a cognitive agent can perceive environmental states $x_{E}^{t}$ and internal states $x_{A}^{t}$, perform decision behaviors $a^{t}$, and receive action reward $r^{t}$ from the cognitive space. Here, we suppose that the $r^{t}$ value should be ultimately evaluated by the cognitive agent itself. Moreover, the pattern representation transforms $\left\{\Phi_{k}\right\}$ and decision policies $\left\{<\Phi_{l}, a_{l}, u_{l}, \hat{r}_{l}>\right\}$ are all internal components of a cognitive system. They may be gradually evolved by adapting to its surviving environment. So, there exists implied information exchange between the inner and the outer of a module denoted by dash lines.

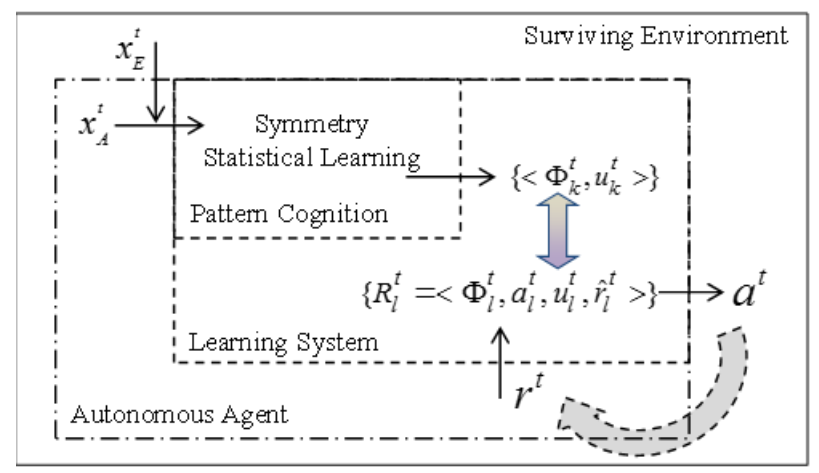

Fig. 2. Evolutionary learning process of EEL

The above evolutionary learning process contain three core parts: getting new decision experience according to latest states of the cognitive space, updating utility probability values of the current decision policies, and adjusting pattern representation and decision policies. Some detailed implementations will be described below.

In this study, the symmetry representation is characterized by relation tuples $<\Phi_{l}^{t}\left(x^{t}\right), a_{l}^{t}, r_{l}^{t}>$ and $\Phi_{k}^{t}=<\phi_{j}\left(\left\{x^{t}\right\}_{t \leq T}, \theta_{j}\right)>_{j \in C_{k}}$. We assume that all symmetry relations could be extracted in advance by artificially analyzing causalities existing in the cognitive space. Furthermore, for the satisfaction of computability, all causalities are divided into two types: complete or incomplete. For a given decision policy, the complete causality means that, optimal actions and corresponding expectation rewards can be completely determined by the input. By contrast, input information in a decision policy is insufficient to decide an optimal action for an incomplete case. No doubt that incomplete cases may be more common and widely useful in many practical applications especially in random systems. For example, first-order Markov processes are usually considered to approximately model conditional causalities in which true causalities may be very complicated [53,54]. In this study, we consider that all necessary symmetry relations could be pre-defined, and further research on how to adaptively learn them will be our future work.

Next, because a decision policy $R_{l}=<\Phi_{l}, a_{l}, u_{l}, \hat{r}_{l}>$ relates to a valid pattern object $\Phi_{l}$, then pattern representation learning has to be jointly done together with decision policy learning. The following learning steps are carried out: 1) newly perceived action rewards $r^{t+1}$ and cognitive space states $x^{t+1}$ are used to construct one new experience $<\Phi^{t+1}\left(x^{t+1}\right), a^{t+1}, r^{t+1}>$ by means of given symmetry representation model. 2) new reference decision rewards $r_{l}^{t+1}$ are evaluated for every leant decision policies to form $<R_{l}^{t}, r_{l}^{t+1}>$. 3) decision policies $\left\{R_{l}^{t+1}\right\}$, pattern representation $\Phi_{k}=<\phi_{j}\left(\bullet, \theta_{j}\right)>_{j \in C_{k}} \quad$ and $\Phi=\left\{<\Phi_{k}, u_{k}>\right\}$ are jointly optimized by means of utility-selection learning strategy.

In summary, the proposed framework makes it feasible to guide the joint learning of pattern representation and decision policies only using decision reward as supervised information. Clearly, the above learning process is considerably different from existing traditional machine learning methods. Specifically, the utility-selection learning strategy is introduced to solve explicit learning of pattern representation and decision policies.

\section{Core algorithms}

In this subsection, we will introduce the core algorithms necessary for implementing the framework of EEL. According to the above discussions, all required symmetry relationships could be pre-defined. Here, the learning updating could be triggered when a new experience item $<\Phi^{t}\left(x^{t+1}\right), a^{t+1}, r^{t+1}>$ is acquired. For the updating of the expectation reward $\hat{r}_{l}^{t+1}$ in $R_{l}^{t+1}$, we directly borrow the updating strategy of Q-value function $Q^{t+1}\left(\Phi_{l}^{t+1}, a_{l}^{t+1}\right)$ designed in reinforcement learning. Moreover, Inspired by the process of human decision development [55], utility-selection learning strategies will be used to progressively improve decision-making and pattern representation. Several studies in experimental psychology by Rieskamp [55] indicated that human participants could use strategy selection theory (SSL) to gain nearly the best predictions after sufficient reinforcement learning loops. Next, based on utility-selection learning strategy, several core algorithms of EEL are discussed.

\section{Utility value updating}

In EEL, utility probability values are introduced to guide memory selection of pattern representation and decision policies. In principle, those items with higher utility probability values should be preferentially stored. On the 
contrary, those items with low utility probability values may be forgotten or replaced by new useful item. The utility probability value updating equations for pattern items and decision policy items can be defined as follows, respectively.

$$
u_{k}^{t+1}=\lambda_{1} \times u_{k}^{t}+\mu_{1}\left(<\Phi^{t}\left(x^{t+1}\right), a^{t+1}, r^{t+1}>, \Phi_{k}^{t}\right)
$$

and

$$
u_{l}^{t+1}=\lambda_{2} \times u_{l}^{t}+\mu_{2}\left(<\Phi^{t}\left(x^{t+1}\right), a^{t+1}, r^{t+1}>, R_{l}^{t}\right)
$$

where $\lambda_{1}, \lambda_{2} \in[0,1)$ are forgetting factors. Furthermore, we may consider $\mu_{1}\left(<\Phi^{t}\left(x^{t+1}\right), a^{t+1}, r^{t+1}>, \Phi_{k}^{t}\right)=\mu_{1}\left(r^{t+1}\right)$ if $\operatorname{dist}\left(\Phi^{t}\left(x^{t+1}\right), \Phi_{k}^{t}\left(x^{t+1}\right)\right)$ is the minimum distance for all pattern items in $\left\{\Phi_{k}^{t}\right\}$ and it is less than $\varepsilon_{1}$, otherwise $\mu_{1}\left(<\Phi^{t}\left(x^{t+1}\right), a^{t+1}, r^{t+1}>, \Phi_{k}^{t}\right)=0$. Similarly, we may consider that $\mu_{2}\left(<\Phi^{t}\left(x^{t+1}\right), a^{t+1}, r^{t+1}>, R_{l}^{t}\right)=\mu_{2}\left(r^{t+1}\right)$ if $\operatorname{dist}\left(<\Phi^{t}\left(x^{t+1}\right), a^{t+1}>,<\Phi_{l}^{t}\left(x^{t+1}\right), a_{l}^{t}>\right)$ is the minimum distance for all decision policies in $\left\{R_{l}^{t}\right\}$ and it is less than $\varepsilon_{2}$, otherwise, $\mu\left(<\Phi^{t}\left(x^{t+1}\right), a^{t+1}, r^{t+1}>, R_{l}^{t}\right)=0 \quad$. In practice, we may consider $\mu_{1,2}\left(r^{t+1}\right)$ equal to constants or $r^{t+1}$. Thus, the utility probability value may be viewed as a discounted cumulant on the historical usefulness of a memory item.

\section{Decision policy learning algorithm}

Here, we discuss how to update decision policies based on utility-selection learning strategy. When the number of storable decision policy items denoted by $N_{R}$ is bounded, there will exist two type of possible updating cases: 1) slightly adjusting parameters of certain decision policy; 2) replacing an old policy with newly generated policy. Here, $\varepsilon$-approximation is used to decide whether newly coming experience $<\Phi^{t}\left(x^{t+1}\right), a^{t+1}, r^{t+1}>$ could be integrated into existing decision policies. More generally, we employ evolutionary sampling learning proposed in [48] to implement utility-selection learning for decision policy updating.

Let the approximation objective $\pi^{t}(\bullet) \equiv u_{l}^{t+1}(\bullet)$ and introduce $p^{t}(\bullet)$ defined as follows

$$
p_{l}^{t}=\sum_{j}\left(\exp \left(-\operatorname{dist}_{R}^{2}\left(R_{l}^{t}, R_{j}^{t}\right) / \sigma_{R}\right) \times u_{j}^{t}\right)
$$

where, $\operatorname{dist}_{R}(\bullet, \bullet)$ denotes the distance between two decision policies, $\sigma_{R}>0$ is a scalar parameter, and $\exp (\bullet)$ is the exponential function. So, $\exp \left(-\operatorname{dist}_{R}^{2}\left(R_{l}^{t}, R_{j}^{t}\right) / \sigma_{R}\right)$ could be viewed as a correlation coefficient between two decision policies. Then, the evolutionary sampling learning objective is defined as to make $p^{t}(\bullet) \rightarrow \pi^{t}(\bullet)$ on all decision policies. In addition, let $p_{n}^{t}$ and $\pi_{n}^{t}$ denote the evaluated and expected utility probability values of newly acquired experience $<\Phi^{t}\left(x^{t+1}\right), a^{t+1}, r^{t+1}>\quad$ respectively, in which $\pi_{n}^{t}=u_{n}^{t+1}=\mu_{2}\left(r^{t+1}\right)$.

In summary, following algorithm is proposed for decision policy learning.

\section{Decision Policy Learning Algorithm in Evolutionary \\ Enactive Learning}

1 Given a distance measure on the pair $\left\langle\Phi_{l}^{t}, a_{l}^{t}\right\rangle$, prior parameters $\varepsilon$, and $N_{R}$, let decision policy set $\Re^{t}=\left\{R_{l}^{t}\right\}=\varnothing$ in which $t=0$.

2 For every newly generated experience $R_{n}^{t+1}=<\Phi_{n}^{t}\left(x^{t+1}\right), a_{n}^{t+1}, r_{n}^{t+1}>$, perform steps 3-8.

3 If $\left|\Re^{t}\right|<N_{R}, \Re^{t+1}=\mathfrak{R}^{t} \cup R_{n}^{t+1}$ and go to step 2; otherwise, go to next step.

4 Find the decision policy item $R_{o}^{t}$ in $\mathfrak{R}^{t}$ that has the minimal distance to $R_{n}^{t+1}$, then compute $u_{n}^{t+1}=\mu_{2}\left(r^{t+1}\right)$.

5 If $\operatorname{dist}_{R}\left(R_{o}^{t}, R_{n}^{t+1}\right)<\varepsilon$, update $R_{o}^{t} \quad$ using statistical combination between $R_{o}^{t}$ and $R_{n}^{t+1}$, and go to step 2; otherwise, go to next step.

6 Re-choose the decision policy item $R_{o}^{t}$ in $\mathfrak{R}^{t}$ with minimum $\pi_{o}^{t}$, then compute $p_{o}^{t}, p_{n}^{t}$, $\pi_{n}^{t}$.

7 Get a uniform random number $z$ on $[0,1]$; if $z<\min \left\{1, \frac{\pi_{n}^{t}}{\pi_{o}^{t}}\right\}$ receive $R_{n}^{t+1}$ provisionally, then go to the next step; otherwise exit learning loop and go to step 2 .

8 Generate another uniform random number $z^{\prime}$ on $[0,1]$, if $z^{\prime}<\max \left\{0,1-\frac{\pi_{o}^{t}}{\pi_{n}^{t}} \times \frac{p_{n}^{t}}{p_{o}^{t}}\right\}$, replace $R_{o}^{t}$ with $R_{n}^{t+1}$ in $\mathfrak{R}^{t}$ to form $\mathfrak{R}^{t+1}$.

\section{END}

In above decision policy learning algorithm, the decision policy replacing strategy originates from evolutionary sampling learning approach [48], which enables the decision policies to strike a good balance between different possible options when the number of policy items to be stored is limited. Moreover, this strategy may produce two benefits: discarding those decision policies with small utility probability values and preserving high diversity among different decision policies. In addition, the function $\operatorname{dist}_{R}(\bullet, \bullet)$ and the statistical combination strategy on $R_{o}^{t}$ and $R_{n}^{t+1}$ should be extra designed for practical applications.

\section{Pattern representation learning}

In EEL, we use utility probability value $u_{k}$ to determine whether a possible pattern representation $\Phi_{k}$ should be stored in the cognitive agent's memory. For most cases, we may select top $N_{P}$ items with the highest utility probability values if the number of storable pattern representation items $N_{P}$ is limited. This will be our default consideration in this study and could be viewed as a simple variant of the strategy considered in classical 
reinforcement learning methods, where all possible pattern representation items are as possible as memorized. Nevertheless, evolutionary sampling learning presented in our previous study [48] also could be employed to subtly keep most useful pattern representation items like the decision policy learning algorithm presented above. Finally, existing unsupervised pattern representation learning could also be integrated into the framework of EEL to offer more flexible learning abilities.

\section{Decision selection algorithm}

Here, we further discuss the problem how to use decision policy to choose behavior actions. When input states are continuous, there may be no exact match between new input observation $\Phi_{o b}^{t+1}$ and pattern representation items in $\left\{\Phi_{l}^{t}\right\}$. Usually, there will exist multiple optional decision policies partially suited for the current input. Inspired by statistical inference theory, the statistical decision output strategy is presented. And the following weight value evaluation for every decision policy is designed.

$$
w_{l}^{t}=\exp \left(-d i s t_{\Phi}^{2}\left(\Phi_{o b}^{t+1}, \Phi_{l}^{t}\right) / \sigma_{\Phi}\right)\left(u_{l}^{t}\right)^{\gamma},
$$

where, $\gamma \in(0, \infty)$ is a deformation factor, $\operatorname{dist}_{\Phi}(\bullet, \bullet)$ denotes the distance between two pattern representation items, $\sigma_{\Phi}$ is a scalar factor. In particular, $\operatorname{dist}_{\Phi}(\bullet, \bullet)$ may only output $\infty$ or 0 for the cases where only exact input match is valid.

Thus, we consider $w_{l}^{t}$ as a relative probability value to choose corresponding decision policy $\boldsymbol{R}_{l}^{t}$. Moreover, we propose the following decision selection algorithm used in evolutionary enactive learning.

\section{Statistical Decision Output Algorithm in Evolutionary Enactive Learning}

1 Given a distance measurement on $\left(\Phi_{o i}, \Phi_{l}\right)$, and a current decision policy set $\mathfrak{R}^{t}=\left\{R_{l}^{t}\right\}$.

2 For current observed input $\Phi_{o b}\left(x^{t+1}\right)$, compute $w_{l}^{t}$ for every feasible policy.

3 Randomly choose a decision policy $\boldsymbol{R}_{c}^{t}$ with probability $\frac{w_{c}^{t}}{\sum_{l} w_{l}^{t}}$.

4 Output the action of $R_{l}^{t}$ as the current decision action.

\section{END}

Equation (9) is compatible to the cases that multiple actions are available for the same input state, especially in case of finite discrete input states. In addition, the observation $\Phi_{o b}^{t+1}$ may be information-incomplete. That is, $\Phi_{l}^{t}$ in $R_{l}^{t}$ should contain more information to make an absolutely credible decision. In addition, some variable definitions on $\operatorname{dist}_{\Phi}(\bullet, \bullet)$ may be allowed for practical problems.

\section{Theoretical discussions}

The proposed EEL framework aims to realize a newly autonomous development framework of cognitive capabilities. Theoretical performance analysis on EEL is provided in Appendix A.

As a preliminary instantiation of the basic idea in enactive AI, the evolutionary mechanism of EEL mainly contains the following three aspects: 1) two persistent memory sets (pattern representation set and decision policy set), which are the representation of the solutions; 2) the utility maximum priority criterion, which is designed as the objective of the evolutionary optimization; 3) evolutionary sampling learning strategy employed as the search and selection strategies of the evolutionary optimization.

Compared to DRL [11], EEL has strong capability of learning explicit pattern and policy representation, which is also motivated from enactive AI model. On top of this, several new evolutionary mechanisms are designed as described above. Consequently, EEL can take into account of not only complex sensory inputs as in DRL, but also a huge number of optional actions as in [12]. In addition, deep representation networks designed in DRL is also available in EEL to model highly complex observation inputs. In summary, EEL could be seen as attempt to imitate the developmental process of human cognitive control. It should be mentioned that the decision policy learning algorithm developed in EEL can be extended to DRL to optimally select a small number of experience tuples.

Compared to other existing RL methods, EEL aims to realize more flexible and explicit (white-box) pattern and policy representation learning framework envisioned in enactive AI [17]. Note that for problems that can be well solved by traditional RL, EEL does not necessarily have advantage in performance.

In addition, as a generic learning process, EEL is open to extensions and specialization for different practical applications. For example, the distance measure methods on pattern items and decision policy items, and combination of representation forms for abstract pattern, and pattern item memory strategy should be flexibly defined in practical applications.

\section{EXPERIMENTAL STUDIES}

\section{A. Simulation scenarios}

In this part, two simulated cognitive decision-making scenarios are designed to examine the performance of EEL. Scenario $\mathrm{I}$ is a color stripe sequence cognition game and Scenario II is an optimal coverage selection game. Note that the cognitive space of Scenario I is discrete while that of Scenario II is continuous. Scenario I is constructed by abstracting the main logic rules used in the building blocks game. Scenario II is inspired by skill learning in agricultural planting, in which some natural laws must be considered. For example, the seasons change periodically, the crop yield may be related to the seasonal temperatures, and only one effective planting may be allowed in one year.

In the two simulated cognitive tasks, we assume that an intelligent agent is able to develop its cognitive behaviors by continuously perceiving the environmental states, making cognitive decisions, and receiving rewards. 
Color stripe sequence cognition game

The color stripe sequence cognition game is illustrated in Fig. 3. In this game, a pattern is represented as a sequence of color stripes with certain sequence. The bottom-left part in Figure 3 shows 20 such sequence patterns with different sequence lengths.

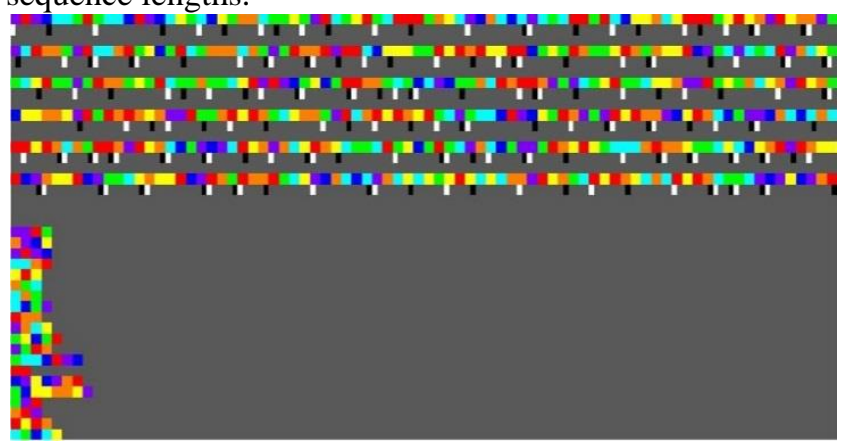

Fig. 3 An illustration of color stripe sequence cognition game

The rules of the game are described as follows. An intelligent agent can continuously receive sequenced color stripes generated by a random strategy. At any time, it can select a group of consecutive color stripes with a certain length from the current observed scope, and then submit the selected sequence to get an evaluation reward. The top half part in Fig. 3 shows a sequence of color stripes continually generated by randomly jointing true color stripe pattern sequences and noisy color stripes. In Fig. 3, some small white and black blocks below the original color stripes are plotted to visualize the starts and ends of true color stripe pattern sequences.

Thus, the cognitive space of the color stripe sequence cognition game comprises the following three components: a group of true color stripe sequence patterns, positive evaluation rewards for any cognitive submission, and the rule of generating observable sequenced color stripes. The evaluation reward for every submission is 1 if the submitted color stripe sequence is identical to the embedded true pattern sequence; otherwise, the reward value is 0 .

The cognitive learning objectives include: 1) to find as many true sequence patterns as possible, and 2) to pick out as many as possible correct pattern sequences embedded in observed sequenced color stripes. In addition, we assume that when a submission is performed, all color stripes before submission point will disappear and cannot be used again. Thus, the previous decision submissions will affect future decisions. Note that the above two cognitive tasks may be slightly conflicting with each other.

In addition, 7 different colors are used to define color stripes, and the length of each color stripe pattern sequence ranges from 2 to 9 . The total number of different true sequence patterns is denoted by $N_{C}$, and the total number of true sequence patterns in once simulation is denoted by $N_{L}$.

\section{Optimal coverage selection game}

In this simulated cognitive game, we assume an agent can perceive an one-dimensional time series, in which one real number can be observed at one time instant. It is expected in the game that an agent can perform optimal coverage selection on every peak zone, in which only once coverage selection will be effective for one peak period.

At first, we employ variable $z(t)$ of the following standard Lorenz chaos system to simulate one-dimensional time series:

$$
\begin{aligned}
& \frac{d x}{d t}=\sigma(y-x) \\
& \frac{d y}{d t}=x(\rho-z)-y \\
& \frac{d z}{d t}=x y-\beta z
\end{aligned}
$$

where $\sigma=10, \rho=28, \beta=\frac{8}{3}$, and initial states $\left[\begin{array}{lll}x & y & z\end{array}\right]_{t=0}=\left[\begin{array}{lll}0 & 1 & 1.05\end{array}\right]$. The sampling interval between two subsequent time points is set as $\Delta t=0.005$ (called as a time interval).

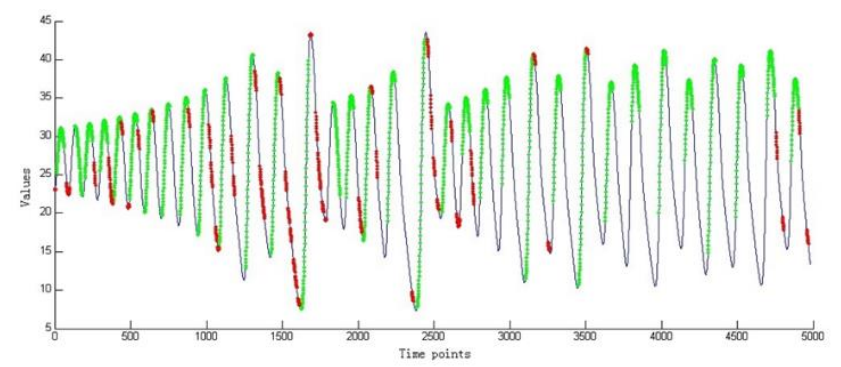

Fig. 4 An illustration of the optimal coverage selection game

Fig. 4 shows a segment of the simulated value curve with a sequence of coverage selection results being attached, in which green segments denote effective coverage selection results while red segments denote failed coverage selection results. For the above periodic curve, we consider that a local varying period starts at one valley point (a local minimal point) and ends at its sequential valley point. Moreover, we call the left half part of a local varying period as up half-period, and the right half part of a local varying period as down half-period. In the following we will introduce the rules of the game in detail.

1) An agent can start a coverage selection at the current time instant if there is no unclosed previous selection, and will get rewards after some time instants for once operation. Once effective coverage selection must start from the up half-period. Otherwise, coverage selection becomes failing and no positive rewards will be given.

2) If once coverage selection is effective, then we set the coverage range as 50 time intervals. If one coverage selection is failing, the coverage range is set to 10 intervals. That is to say, if an agent performs a correct coverage selection, then it can gain positive rewards after 50 time intervals. By contrast, the agent will be able to perceive the effect of a failing selection after 10 time intervals. Some effective or failing selections' results indicated by green or red color, respectively, are illustrated in Fig. 4. 
3) For one effective coverage selection $c_{i}$, the reward is defined as

$$
\operatorname{Rwd}\left(c_{i}\right)=\sum_{j=1}^{50} Z\left(T c_{i}(j)\right)
$$

where $T c_{i}(j)$ denotes the $j$ th time instant after the coverage start of coverage selection $c_{i} \cdot Z(\bullet)$ is the variable value with respect to every time instant.

For this game, the developmental self-intention of individual cognitive evolution aims to get as much reward as possible and as less failing selection as possible. This is however, under the constraint that only limited memory capacity for storing experience is available to the agent. Usually, there exists one optimal coverage start point for every local varying period and doing one effective selection for one local period only is the best.

\section{Discussions}

In the two scenarios, we introduce more realistic considerations for real-world problem solving, including requirements on flexible and explicit pattern/policy representation and unknown environmental changes. DRL or existing RL methods will have difficulties in modeling these two problems. For example for scenario I, it will be very difficult for DRL to construct balanced training sample set without an explicit pattern representation learning mechanism. Besides, DRL is not able to extract explicit pattern representation, which does not satisfy with the problem requirements. Although the classical RL methods do not have the above issues they are very likely subject to combinatorial rule explosion problem.

Solving scenario II requires open system states prediction cognition, where decisions need to be made in the presence of uncertainty. Existing RL methods have no joint learning strategies for state prediction in the presence of uncertainty and policy decision required in enactive AI.

By contrast, EEL is equipped with several newly added mechanisms including flexible and explicit pattern/policy representation, and a utility-selection learning strategy, which are essential requirements in enactive AI.

\section{B. EEL algorithm settings for the two scenarios}

As previously indicated, EEL is a generic learning framework for modeling collaborative cognitive development learning. To apply EEL to the two cognitive learning tasks discussed above, problem-specific settings need to be defined. Mainly, three modules: pattern representation learning, decision action selection, and decision policy learning should be implemented in detail for above two cognitive learning problems.

\section{Settings for the color stripe sequence cognition game}

In the EEL framework, we use tuples $\left\{<\Phi_{k}, u_{k}>\right\}$ to model pattern representation learning, and those pattern representation items with high utility values should be memorized with a higher priority. Here, the ground pattern representation is denoted by $\phi_{j}, j=1,2, \cdots, 7$, in which the total number of possible color stripes is 7 . And a pattern $\Phi_{k}$ is autonomously constructed by combining another pattern $\Phi_{k j}$ and a ground pattern. That is $\Phi_{k}=<\phi_{j}>$ or $\Phi_{k}=<\Phi_{k j}, \phi_{j}>$, in which it might be possible that $\Phi_{0}=\{\}$. In addition, only two possible actions, either to submit or not to submit, can be performed for every observed pattern sequence. Thus, the representation model of decision polies is fully complete according to the definition I in Appendix A.

Moreover, $u_{k}$ could be defined as follows.

$$
u_{k}(t+1)=\lambda_{1} \times u_{k}(t)+I S_{k}(t+1) \times\left(1-\lambda_{1}\right)
$$

where $\quad I S_{k}(t+1)=\left\{\begin{array}{l}1, \text { perceived } \\ 0, \text { otherwise }\end{array} \quad\right.$ indicates $\quad$ whether pattern item $\Phi_{k}$ is perceived at current time instant $t$ or not. The forgetting factor $\lambda_{1}=0.995$ is used in our simulations.

For the decision policies updating, we assume that $\mu\left(<\Phi^{t}\left(x^{t+1}\right), a^{t+1}, r^{t+1}>,<\Phi_{l}^{t}, a_{l}^{t}>\right)$ equals $\left(1-\lambda_{2}\right)$ or 0 for effective or failing decision submission, respectively. Thus, we can define that

$$
u_{l}(t+1)=\left\{\begin{array}{l}
\lambda_{2} \times u_{l}(t)+I u_{l}(t) \times\left(1-\lambda_{2}\right), \text { do submit } \\
u_{l}(t), \text { donot submit }
\end{array}\right.
$$

where $I u_{l}(t)=\left\{\begin{array}{l}1, \text { effective decision } \\ 0, \text { failing decison }\end{array}\right.$ indicates whether the decision is effective or failing. The updating factor $\lambda_{2}=0.6$ is adopted by our experimental comparisons in this study. Naturally, $u_{l}(0)=0.5$ could be set as the default value for any sequence pattern items. It is easy to see $u_{l}(t) \in(0,1)$ for any $t$. In addition, we assume that different pattern sequences are uncorrelated, so $\exp \left(-\right.$ dist $\left._{\Phi}^{2}\left(\Phi_{o b}^{t+1}, \Phi_{l}^{t}\right) / \sigma_{w}\right)$ should be set to 1 if $\Phi_{o b}^{t+1} \equiv \Phi_{l}^{t}$, or 0 if not. Accordingly, $w_{l}^{t}=\left(u_{l}^{t}\right)^{0.5}$ is considered according to Appendix A. Thus, if one observed color stripe sequence matches the conditional pattern of a decision policy, the cognitive submission may be determined with a probability $\frac{\left(u_{l}^{t}\right)^{0.5}}{\left(u_{l}^{t}\right)^{0.5}+\left(1-u_{l}^{t}\right)^{0.5}}$. If more than one pattern sequences in decision policies could be matched at current observation, we may select the one having the highest $u_{k}^{t}$ as the candidate decision policy.

\section{Settings for coverage selection game}

For the optimal coverage selection game, several parts of settings also need to be specified include: pattern representation learning, coverage selection decision policy learning, and decision output strategy.

For the pattern representation learning, a ground pattern item $\phi_{j}$ is used to express a variable changing pattern of an up or down half-period, and for simplicity, is denoted as 
a two-dimensional vector (slope, duration) ${ }_{j}$, where, "slope" denotes the average changing gradient, and "duration" the time length of the corresponding half-period. Moreover, an abstract pattern $\Phi_{k}$ can be represented by a ground pattern or the combination of tuples $<\phi_{k 1}, \phi_{k 2}, \phi_{k 2}>$. $u_{k}$ in $\left\{<\Phi_{k}, u_{k}>\right\}$ is defined as follows:

$$
u_{k}(t+1)=\lambda_{1} \times u_{k}(t)+I S_{k}(t+1) \times\left(1-\lambda_{1}\right)
$$

where, $I S_{k}(t+1)=\left\{\begin{array}{l}1, \text { perceived } \\ 0, \text { otherwise }\end{array}\right.$, and $\lambda_{1}=0.995$ is adopted from our experimental comparisons. Moreover, the distance metric on two pattern items is defined by

$$
\operatorname{dist}\left(\Phi_{1}, \Phi_{2}\right)=\max \left(\frac{a b s\left(\Phi_{1}-\Phi_{2}\right)}{a b s\left(\Phi_{1}\right)+a b s\left(\Phi_{2}\right)}\right)
$$

where, $\Phi_{1}, \Phi_{2}$ are two vectors, function $a b s(\bullet)$ returns a new vector by getting the absolute values of the input vector's values in each dimension, and function $\max (\bullet)$ outputs the maximum value of a vector. In addition, $\exp \left(-\right.$ dist $\left._{\Phi}^{2}\left(\Phi_{o b}^{t+1}, \Phi_{l}^{t}\right) / \sigma_{\Phi}\right)$ is simplified to be 1 or 0 according to $\varepsilon$-approximate criterion, where $\varepsilon=0.1$ is used according to our experimental analysis.

For coverage selection policy learning, the decision policies are described as the tuple $<\Phi_{l}\left(\phi_{l 1}, \phi_{l 2}, \phi_{l 3}\right), a_{l}, u_{l}, \hat{r}_{l}>$ in terms of the framework of EEL, where $\Phi_{l}$ is the combination of three consecutive ground pattern items $\phi_{l 1}, \phi_{l 2}, \phi_{l 3}, a_{l}$ is an optimal coverage selection decision vector related to $\Phi_{l}, u_{l}$ is the decision utility probability value, and $\hat{r}_{l}$ denotes corresponding weighted reward according to historical experiences. Here, we define

$$
u_{l}(t+1)=\lambda_{2} \times u_{l}(t)+\hat{r}_{l}(t+1)
$$

and

$$
\hat{r}_{l}(t+1)=\beta \times \hat{r}_{l}(t)+(1-\beta) \times \mu_{R w d}(t+1)
$$

where, $\mu_{R w d}(t+1)$ represents the reward from the current valid coverage selection experience $<\Phi^{t+1}, a^{t+1}, \mu_{R w d}(t+1)>$ at time $t+1$. We set $\lambda_{2}=0.995$, $\beta=0.99$ and $\hat{r}_{l}(1)=\mu_{l}\left(t_{0}\right)$ by our experimental analysis, where $t_{0}$ is the time instant when the rule $R_{l}$ was created. We use the relaxed distance threshold $\varepsilon=0.35$ on $\Phi$ to determine whether a near decision policy $R_{l}$ is updated based on new experience $<\Phi^{t+1}, a^{t+1}, \mu_{R w d}(t+1)>$.

Decision variable $a_{l}$ should also be gradually optimized for this cognitive problem. Here, we assume $a_{l}$ to be a 30 dimensional vector, in which each element denotes a probability of starting coverage selection at a related time instant within the time covered by $\Phi_{l}\left(\phi_{l 1}, \phi_{l 2}, \phi_{l 3}\right)$. Next, the following action optimization strategy is introduced:

$$
\operatorname{prob}\left(a_{l}, j, t+1\right)=\left\{\begin{array}{l}
\operatorname{prob}\left(a_{l}, j, t\right) \times 1.2, \text { effective selection } \\
\operatorname{prob}\left(a_{l}, j, t\right) \times 1.0, \text { no selection } \\
\operatorname{prob}\left(a_{l}, j, t\right) / 1.2, \text { failing selection }
\end{array}\right.
$$

where $j \in\{1,2, \cdots, 30\}$, and 30 intervals are uniformly distributed in the range related to $\Phi_{l}$.

In addition, the representation form used in coverage selection policies must involve unknown future states. Here, the partial distance metric match is considered between the conditional pattern of decision policies and current observation, and the subpart $\left\langle\phi_{l 1}, \phi_{l 2}\right\rangle$ of $\Phi_{l}$ is used to compute the match degree between a decision policy and the current observation. Moreover, if more than one decision policy is matched, we choose the one with the maximum $u_{l}$ as the referenced policy.

\section{Experimental results}

\section{Results on the color stripe sequence cognition game}

In this subsection, the experimental results on color stripe sequence cognition game will be reported. For comparison, two random cognitive strategies, greedy random cognition (GRC) and balanced random cognition (BRC) will be introduced before we discuss the experimental result.

The GRC learning strategy first seek whether there is a pattern sequence that can be matched to stored pattern sequences. If there is a match, that matched color stripe sequence will be submitted; otherwise, a pattern sequence ranging from 2 to 9 in latest observation will be randomly select to explore new possible color stripe pattern. The BRC learning strategy uses a similar random searching strategy, but it does not always submit matched color stripe sequence. We set the submission probability as 0.8 to balance the requirement of exploring new possible patterns and picking out known patterns. EEL will automatically create all new possible pattern items according to the latest observations. The earlier a pattern is stored, the lower the utility value $u$ will be. In our simulations, limited storable pattern items $N_{P}=200$ is set as default, which we found sufficient in our experiments.

In all simulations, we set the number of different true pattern sequences $N_{C}=40$, in which the sequence length ranges from 2 to 9 . The ratio between the numbers of two types of sequences with lengths of $i+1$ and $i$ is set as 0.85. Between two embedded true patterns, noisy sequences are added with a probability of $50 \%$. For those sequences with noise being added, the noisy stripe sub-sequence length ranges from 1 to 5 , and possible color stripes are uniformly selected from 7 different color stripes.

Three performance evaluation indices are adopted in the comparisons. The index "discovery ratio" indicates the ratio of the number of totally discovered true patterns to $N_{C}$. The index "recall ratio" denotes the ratio of successfully picked true patterns, and the index "precision" indicates the correct ratio of cognitive submissions.

Table II lists the simulation results obtained by GRC, $\mathrm{BRC}$ and EEL. The simulation results are averaged over 50 
independent runs.

TABLE II

PERFORMANCE RESULTS OBTAINED BY EEL AND COMPARED METHODS ON PROBLEM I WITH $N_{L}=20000$ AND $N_{C}=40$

\begin{tabular}{llll}
\hline & $\begin{array}{l}\text { Discovery } \\
\text { ratio(\%) }\end{array}$ & $\begin{array}{l}\text { Recall ratio } \\
(\%)\end{array}$ & $\begin{array}{l}\text { Precision } \\
(\%)\end{array}$ \\
\hline GRC & $78.45 \pm 6.85$ & $30.44 \pm 3.28$ & $42.05 \pm 7.85$ \\
BRC & $93.75 \pm 3.98$ & $25.15 \pm 2.69$ & $42.60 \pm 7.63$ \\
EEL & $\mathbf{9 8 . 2 0} \pm \mathbf{2 . 1 8}$ & $\mathbf{5 4 . 1 1} \pm \mathbf{5 . 0 4}$ & $\mathbf{5 9 . 3 2} \pm \mathbf{3 . 4 8}$ \\
\hline
\end{tabular}

The results in Table II demonstrate that EEL has achieved significantly better cognitive performance compared to the two common random cognitive strategies. By comparing the results obtained by GRC and BRC, we find that there is a negative correlation between the performance of discovery ratio and recall ratio, which is rational. By contrast, EEL can achieve a good balance between exploring new possible patterns and picking out known patterns.

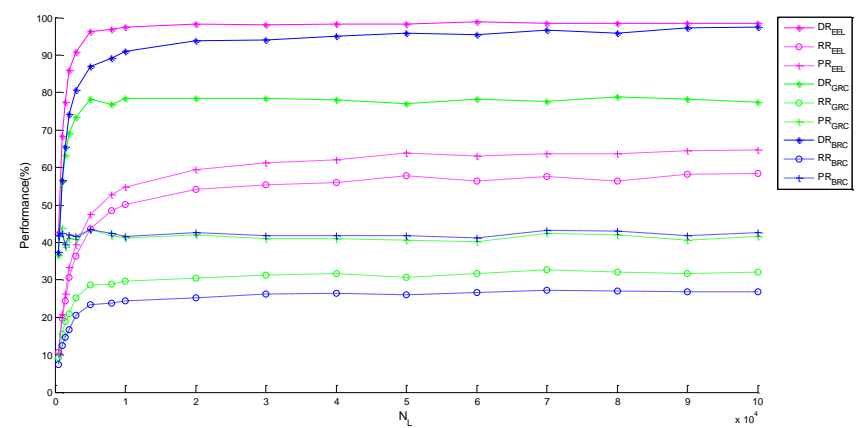

Fig. 5. Performance curves obtained by EEL, GRC, and

$\mathrm{BRC}$ for the color stripe sequence cognition game with different $N_{L}$

Fig. 5 shows a group of performance curves obtained by EEL, GRC and BRC on the color stripe sequence cognition game with different $N_{L}$. In the figure, DR, RR and PR stands for "discovery ratio", "recall ratio", and "precision", respectively. By comparing the performance curves of the three cognitive learning strategies, it is clearly that EEL achieves the more significant improvement than the two random strategies GRC and BRC in terms of the overall cognitive development. The tendency of performance curves of EEL demonstrates that it can progressively improve its own cognitive performance, and converge to a stable value. These experimental results indicate that the learning procedure of EEL is effective for color stripe sequence cognition game.

For BRC and GRC, we also find that, the performance indices "discovery ratio" and "recall ratio" are conflicting with each other. BRC using the balanced random strategy obtains a better discovery ratio but a worse recall ratio, compared to GRC using greedy random strategy. This means that the decision criterion used in the random cognitive strategies are unable to well balance the requirement of exploring new patterns and exploiting old patterns. More adaptive or flexible decision policies must be considered like in human-like cognitive behavior. Naturally, the flexible cognitive decision policies composed of pre-condition, actions and expectation reward originated from standard reinforcement learning might be useful. This consideration also be retained in EEL. In addition, EEL can further improve the flexibility of pattern representation and decision policy learning by combining utility-selection theory and the evolutionary sampling learning strategy. In this sense, EEL can be seen as an extended learning framework compared to traditional reinforcement learning.

In addition, when the maximum length of a true color stripe pattern is limited to 9 , the total number of all possible pattern $N_{C_{-} \text {all }}=\sum_{i=2}^{9} 7^{i}=47079200$, which is a huge value when completely storing them like in classical RL frameworks. Our results indicate that EEL has achieved a significant improvement on cognitive performance compared to the random cognitive strategies by only using $N_{P}=200$ pattern memory items.

\section{Results on coverage selection game}

Like in the first task, we introduce a random strategy algorithm (RSA) for comparison in evaluating the cognitive performance of EEL. In RSA, the probability of starting coverage selection is set to 0.1 according to our pilot studies. The above random strategy will also be used in EEL if no decision policy matches the current observations. Moreover, we set the maximum number of storing pattern items $N_{P}=100$, the maximum number of storing coverage selection decision policies $N_{R_{-} c s}=30$, which have shown to be adequate according to our preliminary studies.

To evaluate the cognitive learning performance, the following two quantitative indices are considered in our simulations. Coverage accuracy (CA) denotes the ratio of effective coverage selection in all selections. Coverage efficiency (CE) denotes the reward efficiency of coverage selection decision strategy. CA and CE can be calculated as follows.

$$
\begin{aligned}
& \mathrm{CA}=N c e \div N c \times 100 \% \\
& \mathrm{CE}=R_{g t c} \div R_{\text {igtc }} \times 100 \%
\end{aligned}
$$

where Nce denotes the effective coverage selection times, $N c$ the total coverage selection times, $R_{g t c}$ the gained total coverage rewards, and $R_{\text {igtc }}$ the ideal total coverage rewards.

Different from the color stripe sequence cognition game, the above two performance indices are independent. Here, "effective coverage times" refers to the total number of effective coverage selection in one simulation, in which the total number times of coverage selection are performed. "Ideal total coverage rewards" refers to the optimal total reward value for one simulation. "gained total coverage rewards" is the sum of the rewards of all effective coverage selections. The ideal values of these performance indices are all $100 \%$. Table III lists the typical experimental results obtained by EEL and RSA on the coverage selection task with $T_{L}=200000$, and corresponding number of periods $N_{\text {periods }}=1271$. In Table III, the average values and standard deviation values of performance indices are calculated using the results from 20 independent simulations. 
TABLE III

PERFORMANCE RESULTS OBTAINED BY EEL AND RSA ON PROBLEM II WITH $T_{L}=200000$

\begin{tabular}{lll}
\hline & $\begin{array}{l}\text { Coverage efficiency } \\
(\%)\end{array}$ & $\begin{array}{l}\text { Coverage } \\
\text { accuracy }(\%)\end{array}$ \\
\hline RSA & $81.99 \pm 0.30$ & $15.96 \pm 0.07$ \\
EEL & $\mathbf{9 1 . 3 9 \pm 0 . 6 7}$ & $\mathbf{5 6 . 8 3} \pm \mathbf{1 . 2 3}$ \\
\hline
\end{tabular}

Similar to the color stripe sequence cognition game, EEL also achieves considerable performance improvement compared to optimal random strategies. EEL has not only improved the coverage selection accuracy more than 2 times, but also achieved higher overall reward. These results indicate that EEL is effective not only in reducing the failing coverage selections but also in improving effective coverage selections with the help of evolutionary cognitive development.

Performance curves for EEL and RSA for the coverage selection task with different $T_{L}$ are plotted in Fig. 6 . Overall, EEL has obtained the best performance, and can converge to stable results as simulation time increases. The performance curves obtained by EEL gradually rises at the beginning and then remains steady. For RSA, its performance curves keep almost constant for different $T_{L}$. These results indicate that the designed cognitive task is statistically stable. The above experimental results also demonstrate the effectiveness of EEL in developing individual cognitive decision behaviors.

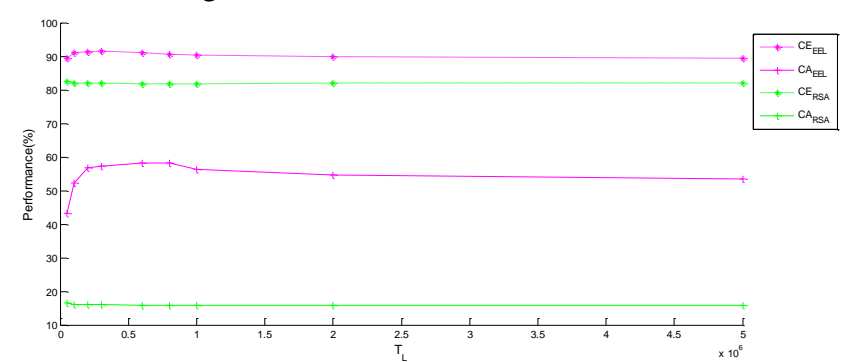

Fig. 6. Performance curves obtained by EEL and RSA on coverage selection with different $T_{L}$

It should be noted that decision actions in the coverage selection task have a huge space and needs to be dynamically optimized, which is very different from classical reinforcement learning. In classical reinforcement learning, all possible actions are considered to be finitely countable, and the total number is not larger than 10 in most studies $[11,13]$. However, action variables of decision policies for coverage selection game designed in this work are continuous. This task is very meaningful extension to traditional reinforcement learning as considered in $[12,14]$. Our experimental results on the coverage selection game task show that EEL is a feasible algorithm for solving such types of RL tasks.

\section{Discussions}

To demonstrate the effectiveness of the proposed EEL, two novel cognitive decision tasks are designed. The color stripe sequence cognition game is motivated by the cognitive combination intelligence that humans usually use in e.g., playing "building blocks" game. The optimal coverage selection game is abstracted from the prediction selection abilities found in intelligent cognitive behaviors. No doubt, the above two types of cognitive tasks are very valuable for studying human-like cognitive learning. Meanwhile, they are inevitable for measuring whether an intelligent machine possesses human-like cognitive developmental learning capabilities.

To the best of our knowledge, existing RL methods are not able to directly model the above two simulated cognition game problems. Therefore, we can only introduce naïve random cognitive algorithms for empirical comparisons. The experimental results reported in this study indicate that EEL is remarkably effective.

It should be mentioned that the traditional reinforcement learning would be sufficient if all useful decision policies are finite and can be fully recorded. However, in the real world, there are many cases where the conditional states and/or actions in decision policies are infinite or huge. In this case, constrained learning strategies have to be adopted due to the limited computational resources $[11,12,14]$. In this study, we investigate the learning strategy of selectively memorizing a bounded number of pattern representation and decision policies, in which utility probability values are introduced to guide selective memory Our theoretical and experimental results demonstrate that the utility-selection learning strategy proposed in this work is very effective and can be complementary to existing RL strategies $[11,12,14]$.

\section{CONCLUSIONS}

A novel machine learning method, evolutionary enactive learning (EEL) was proposed to model collaborative cognitive development process. In the proposed framework, two cognitive tasks, enactive pattern representation and decision-making, are jointly learnt driven by environmental action rewards. Specifically, three important tools, namely, "symmetry representation model", "utility-selection learning strategy", and "statistical decision output strategy" were introduced to realize above targets.

Two cognitive decision tasks were designed to examine the performance of proposed method. Those tasks cannot be nicely solved by existing RL methods. Our experimental results demonstrated that EEL was effective and could significantly enhance the cognitive performance with evolutionary developmental learning.

From our results, two important observations can be made. First, EEL presented in this study expands the applicability of traditional RL methods and provides a novel machine learning framework as an implementation of enactive artificial intelligence. Second, pattern representation learning guided by utility-selection criterion related to decision reward from the individual cognitive space may be a feasible way to design autonomous intelligent systems.

Nevertheless, according to enactive artificial intelligence, an autonomous intelligent system should have the ability of adaptively regulating its own sensorimotor interaction, 
which is still missing from the proposed EEL framework. It will be our future work to extend EEL by adding and evolving decision policies for sensorimotor control. In addition, some extended implementations of EEL may be feasible for practical cognitive development problems. For example, multiple groups of associated decision policies can be defined and jointly evolved. Deep network structure can also be employed to model decision policy and complicated interpretive relationships over ground patterns. These aspects will also be explored in our future research.

\section{ACKNOWLEDGMENTS}

This work is supported by China Scholarship Program for Visiting Scholar, Jiangsu Province Natural Science Foundation of China (BK20130161), National Key Technology Support Program of China (2015BAH54F01), and National Natural Science Foundation of China (61572236).

\section{REFERENCES}

[1] S. J. Gershman, E. J. Horvitz, and J. B. Tenenbaum, "Computational rationality: A converging paradigm for intelligence in brains, minds, and machines," Science, vol. 349 , no. 6245 , pp. 273-278, 2015.

[2] P. Johnson-Laird, S. S. Khemlani, and G. P. Goodwin, "Logic, probability, and human reasoning," Trends in cognitive sciences, vol. 19, no. 4, pp. 201-214, 2015.

[3] B. M. Lake, R. Salakhutdinov, and J. B. Tenenbaum, "Human-level concept learning through probabilistic program induction," Science, vol. 350, no. 6266, pp. 1332-1338, 2015.

[4] A. Clark, "Whatever next? Predictive brains, situated agents, and the future of cognitive science," Behavioral and Brain Sciences, vol. 36, no. 03, pp. 181-204, 2013.

[5] M. T. Jones, Artificial Intelligence: A Systems Approach: Jones \& Bartlett Learning, 2015.

[6] A. Cully, J. Clune, D. Tarapore, and J.-B. Mouret, "Robots that can adapt like animals," Nature, vol. 521, no. 7553, pp. 503-507, 2015.

[7] J. Bongard, and H. Lipson, "Evolved machines shed light on robustness and resilience," Proceedings of the IEEE, vol. 102, no. 5, pp. 899-914, 2014.

[8] R. Pfeifer, M. Lungarella, and F. Iida, "Self-organization, embodiment, and biologically inspired robotics," Science, vol. 318, no. 5853, pp. 1088-1093, 2007.

[9] D. Floreano, A. J. Ijspeert, and S. Schaal, "Robotics and neuroscience," Current Biology, vol. 24, no. 18, pp. R910-R920, 2014.

[10] G. Infantes, M. Ghallab, and F. Ingrand, "Learning the behavior model of a robot," Autonomous Robots, vol. 30, no. 2, pp. 157-177, 2011.

[11] V. Mnih, K. Kavukcuoglu, D. Silver, A. A. Rusu, J. Veness, M. G. Bellemare, A. Graves, M. Riedmiller, A. K. Fidjeland, and G. Ostrovski, "Human-level control through deep reinforcement learning," Nature, vol. 518, no. 7540 , pp. 529-533, 2015.
[12]D. Silver, A. Huang, C. J. Maddison, A. Guez, L. Sifre, G. Van Den Driessche, J. Schrittwieser, I. Antonoglou, V. Panneershelvam, and M. Lanctot, "Mastering the game of Go with deep neural networks and tree search," Nature, vol. 529, no. 7587, pp. 484-489, 2016.

[13] R. S. Sutton, and A. G. Barto, Reinforcement learning: An introduction: MIT press Cambridge, 1998.

[14]K. Doya, "Reinforcement learning in continuous time and space," Neural Computation, vol. 12, no. 1, pp. 219-245, 2000.

[15]M. L. Littman, "Reinforcement learning improves behaviour from evaluative reward," Nature, vol. 521, no. 7553, pp. 445-451, 2015.

[16]J. Schmidhuber, "Deep learning in neural networks: An overview," Neural Networks, vol. 61, pp. 85-117, 2015.

[17] T. Froese, and T. Ziemke, "Enactive artificial intelligence: Investigating the systemic organization of life and mind," Artificial Intelligence, vol. 173, no. 3, pp. 466-500, 2009.

[18]D. Martínez, G. Alenya, and C. Torras, "Relational reinforcement learning with guided demonstrations," Artificial Intelligence, 2015.

[19] Y. Jin, and J. Branke, "Evolutionary optimization in uncertain environments-a survey," IEEE Transactions on Evolutionary Computation, vol. 9, no. 3, pp. 303-317, 2005.

[20]R. Cheng, and Y. Jin, "A social learning particle swarm optimization algorithm for scalable optimization," Information Sciences, vol. 291, pp. 43-60, 2015.

[21]D. Rus, and M. T. Tolley, "Design, fabrication and control of soft robots," Nature, vol. 521, no. 7553, pp. 467-475, 2015.

[22]Y. Jin, H. Guo, and Y. Meng, "A hierarchical gene regulatory network for adaptive multirobot pattern formation," IEEE Transactions on Systems, Man, and Cybernetics, Part B (Cybernetics), vol. 42, no. 3, pp. 805-816, 2012.

[23] M. Reif, F. Shafait, and A. Dengel, "Meta-learning for evolutionary parameter optimization of classifiers," Machine Learning, vol. 87, no. 3, pp. 357-380, 2012.

[24]L. Zhang, and P. Suganthan, "A Survey of Randomized Algorithms for Training Neural Networks," Information Sciences, 2016.

[25] J. C. Bongard, "Evolutionary robotics," Communications of the ACM, vol. 56, no. 8, pp. 74-83, 2013.

[26]Z.-H. Zhou, N. V. Chawla, Y. Jin, and G. J. Williams, "Big data opportunities and challenges: Discussions from data analytics perspectives [discussion forum]," IEEE Computational Intelligence Magazine, vol. 9, no. 4, pp. 62-74, 2014.

[27]A. E. Eiben, and J. Smith, "From evolutionary computation to the evolution of things," Nature, vol. 521, no. 7553, pp. 476-482, 2015.

[28] Y. Jin. "Surrogate-assisted evolutionary computation: Recent advances and future challenges," Swarm and 
Evolutionary Computation, vol. 1, no. 2, pp. 61-70, 2011.

[29]H. Niemann, "Pattern Analysis and Understanding, volume 4 of Springer Series in Information Sciences," Springer, Heidelberg, 1990.

[30]R. Cappelli, and D. Maltoni, "Multispace KL for pattern representation and classification," IEEE Transactions on Pattern Analysis and Machine Intelligence, vol. 23, no. 9, pp. 977-996, 2001.

[31]D. Mumford, "Pattern theory: the mathematics of perception," arXiv preprint math/0212400, 2002.

[32] T. Watanabe, K. Sugawara, and H. Sugihara, "A new pattern representation scheme using data compression," IEEE Transactions on Pattern Analysis and Machine Intelligence, vol. 24, no. 5, pp. 579-590, 2002.

[33]Y. Bengio, A. Courville, and P. Vincent, "Representation learning: A review and new perspectives," IEEE Transactions on Pattern Analysis and Machine Intelligence, vol. 35, no. 8, pp. 1798-1828, 2013.

[34] Y. LeCun, Y. Bengio, and G. Hinton, "Deep learning," Nature, vol. 521, no. 7553, pp. 436-444, 2015.

[35]G. Alain, and Y. Bengio, "What regularized auto-encoders learn from the data-generating distribution," Journal of Machine Learning Research, vol. 15, no. 1, pp. 3563-3593, 2014.

[36] T. Cover, and P. Hart, "Nearest neighbor pattern classification," IEEE Transactions on Information Theory, vol. 13, no. 1, pp. 21-27, 1967.

[37] R. O. Duda, P. E. Hart, and D. G. Stork, "Pattern classification. 2nd," Edition. New York, 2001.

[38] J.-Y. Zhu, J. Wu, Y. Xu, E. Chang, and Z. Tu, "Unsupervised object class discovery via saliency-guided multiple class learning," IEEE Transactions on Pattern Analysis and Machine Intelligence, vol. 37, no. 4, pp. 862-875, 2015.

[39]D. G. Lowe, "Object recognition from local scale-invariant features," Proceedings of the Seventh IEEE International Conference on Computer Vision, vol. 2, pp. 1150-1157, 1999.

[40] T. Ojala, M. Pietikainen, and T. Maenpaa, "Multiresolution gray-scale and rotation invariant texture classification with local binary patterns," IEEE Transactions on Pattern Analysis and Machine Intelligence, vol. 24, no. 7, pp. 971-987, 2002.

[41]R. Goroshin, J. Bruna, J. Tompson, D. Eigen, and Y. LeCun, "Unsupervised feature learning from temporal data," arXiv preprint arXiv:1504.02518, 2015.

[42] J. Yang, A. F. Frangi, J.-y. Yang, D. Zhang, and Z. Jin, "KPCA plus LDA: a complete kernel Fisher discriminant framework for feature extraction and recognition," IEEE Transactions on Pattern Analysis and Machine Intelligence, vol. 27, no. 2, pp. 230-244, 2005.

[43] R. C. Barros, M. P. Basgalupp, A. C. De Carvalho, and A. A. Freitas, "A survey of evolutionary algorithms for decision-tree induction," IEEE Transactions on Systems, Man, and Cybernetics, Part C (Applications and Reviews), vol. 42, no. 3, pp. 291-312, 2012.
[44]L. Rokach, and O. Maimon, Data mining with decision trees: theory and applications: World scientific, 2014.

[45]C. Cortes, and V. Vapnik, "Support-vector networks," Machine learning, vol. 20, no. 3, pp. 273-297, 1995.

[46]G. Huang, S. Song, J. N. Gupta, and C. Wu, "Semi-supervised and unsupervised extreme learning machines," IEEE Transactions on Cybernetics, vol. 44, no. 12, pp. 2405-2417, 2014.

[47] S. Maji, A. C. Berg, and J. Malik, "Efficient classification for additive kernel SVMs," IEEE Transactions on Pattern Analysis and Machine Intelligence, vol. 35, no. 1, pp. 66-77, 2013.

[48]Z. Xie, J. Sun, V. Palade, S. Wang, and Y. Liu, "Evolutionary sampling: A novel way of machine learning within a probabilistic framework," Information Sciences, vol. 299, pp. 262-282, 2015.

[49] O. Sigaud, and A. Droniou, "Towards deep developmental learning," IEEE Transactions on Cognitive and Developmental Systems, vol. 8, no. 2, pp. 99-114, 2016.

[50] J. B. Tenenbaum, C. Kemp, T. L. Griffiths, and N. D. Goodman, "How to grow a mind: Statistics, structure, and abstraction," Science, vol. 331, no. 6022, pp. 1279-1285, 2011.

[51]L. Bottou, "From machine learning to machine reasoning," Machine Learning, vol. 94, no. 2, pp. 133-149, 2014.

[52]C.-F. Juang, T.-L. Jeng, and Y.-C. Chang, "An Interpretable Fuzzy System Learned Through Online Rule Generation and Multiobjective ACO With a Mobile Robot Control Application," IEEE Transactions on Cybernetics, vol. 46, no. 12, pp. 2706-2718, 2016.

[53]K. Etessami, and M. Yannakakis, "Recursive markov decision processes and recursive stochastic games," Journal of the ACM (JACM), vol. 62, no. 2, pp. 11, 2015.

[54] S. Ross, J. Pineau, B. Chaib-draa, and P. Kreitmann, "A Bayesian approach for learning and planning in partially observable Markov decision processes," Journal of Machine Learning Research, vol. 12, no.5, pp. 1729-1770, 2011.

[55]J. Rieskamp, and P. E. Otto, "SSL: a theory of how people learn to select strategies," Journal of Experimental Psychology: General, vol. 135, no. 2, pp. 207-236, 2006. 